\title{
Perfil de articulação da contribuição e geração de conhecimento em Controladoria e Contabilidade Gerencial
}

\author{
Mamadou Dieng \\ https://orcid.org/0000-0002-5990-8019 | E-mail:mamadou04@hotmail.com \\ Diego Dantas Siqueira \\ https://orcid.org/0000-0002-1421-4080 | E-mail:diego.dantas.pb@gmail.com \\ Dêner Matheus da Silva Viana \\ https://orcid.org/0000-0003-0901-4292 | E-mail:denermatheus81@hotmail.com
}

\section{Resumo}

Objetivo: Analisar a articulação da contribuição dos artigos da área de Controladoria e Contabilidade Gerencial, de 2010 a 2016, na perspectiva de Boxenbaum e Rouleau (2011).

Método: Dados referentes a um total de 190 artigos publicados no Congresso Anpcont, na área temática Controladoria e Contabilidade Gerencial, no período de 2010 a 2016, foram coletados e classificados por meio de categorização conforme cada script epistêmico, por meio da análise descritiva.

Resultados: Constatou-se que a perspectiva dominante da pesquisa é do tipo evolução, mostrando que a pesquisa da área segue a perspectiva da filosofia de Karl (1959) e assume que os pesquisadores, na comunidade brasileira, estão envolvidos em uma busca comum e avanço gradual do conhecimento. Este resultado enquadra-se também na perspectiva da ciência normal de Kuhn (1962). Em outras palavras, pode-se afirmar que a forma de articulação utilizada pelos pesquisadores da área de Contabilidade Gerencial no Brasil corresponde à detecção de uma "lacuna" em pesquisas anteriores.

Contribuiçães: $\mathrm{O}$ estudo proporciona uma melhor compreensão do direcionamento da articulação das pesquisas contemporâneas da Contabilidade Gerencial sobre contribuição à pesquisa, evidenciando as abordagens paradigmáticas, teóricas e metodológicas, e destaca qual (ais) alternativa (s) de script de produção de conhecimento prevalece (em) entre os pesquisadores da comunidade científica.

Palavras-Chave: Conhecimento; Contabilidade gerencial; Pesquisa-Scripts. 


\section{Introdução}

Nos últimos 25 anos, o avanço da pesquisa em Contabilidade Gerencial tem sido objeto de painéis de discussão em eventos comemorativos de aniversário de décadas de pesquisas dos dois principais periódicos de contabilidade gerencial do mundo, o primeiro europeu, o Management Accounting Research (MAR) e o segundo norte-americano, o Journal of Management Accounting Research (JMAR). Na Europa, editoriais do Management Accounting Research: 20 years on (Scapens \& Bromwich, 2001); Management Accounting Research: the firts decade (Scapens \& Bromwich, 2010); Management Accounting Research: 25 years on (Bromwich \& Scapens, 2016) vêm apresentando os principais aspectos relacionados ao desenvolvimento da produção de conhecimento científico em Contabilidade Gerencial segundo relatos de pesquisadores especialistas da área. Também, nos Estados Unidos da América, o debate sobre a pesquisa em Contabilidade Gerencial ocorreu na celebração do $25^{\circ}$ aniversário do Journal of Management Accounting Research (JMAR).

Vários assuntos sobre pesquisa em Contabilidade Gerencial foram abordados, por exemplo, as barreiras para a criação de conhecimento na pesquisa em Contabilidade Gerencial (Salteiro, 2015) e o estabelecimento do conhecimento da Contabilidade Gerencial (Shields, 2015), porém o perfil de articulação das pesquisas em Contabilidade Gerencial permanece um foco a ser investigado e carece de evidências empíricas. Neste contexto, investigar o roteiro empregado para gerar conhecimento consolida os estudos epistemológicos já realizados no campo da Contabilidade Gerencial.

No Brasil, desde outubro de 2013, pesquisadores da área de Contabilidade Gerencial se reuniram no I Encontro de Docentes de Contabilidade Gerencial/Controle Gerencial promovido pela Faculdade de Economia, Administração e Contabilidade da Universidade de São Paulo (FEA-USP) para discutir o desenvolvimento desta área no país. Neste contexto, a comunidade científica da área tem realizado esforços para uma reflexão sobre o progresso da produção de conhecimento em Contabilidade Gerencial, particularmente, o status da área de contabilidade gerencial, as dificuldades para o desenvolvimento e possíveis oportunidades para o futuro (Frezatti, Aguiar, Wanderley \& Malagueño, 2015).

Embora estudos anteriores tanto no cenário internacional (Berry, Coad, Harris, Otley \& Stringer, 2009; Helford, Lee, Van der Stede \& Young, 2006;), quanto nacional (Araújo \& Silva, 2010; Cruz, Costa, Espejo \& Almeida, 2011; Frezatti, Nascimento \& Junqueira, 2008; Lunkes, Ripoll Feliu \& Rosa, 2011; Nascimento, Junqueira \& Martins, 2009) tenham revisado as pesquisas da literatura da Contabilidade Gerencial por meio de análises bibliométrica, bibliográfica e epistemológica, nenhum desses levantamentos tratou diretamente a questão relativa à contribuição e das formas alternativas de articulação para produzir conhecimento científico em Contabilidade Gerencial. Por contribuição à pesquisa, entende-se o incremento que a pesquisa pode fornecer aos conhecimentos já adquiridos sobre um determinado assunto. Em nenhum caso, este incremento deve ser imperativamente revolucionário (Berland, Stolowy \& Piot, 2012). 
Apesar de existir na literatura várias formas de analisar a contribuição de uma pesquisa (Croom, 2001; Gendron, 2008), Boxenbaum e Rouleau (2011) apresentam uma estrutura que aborda algumas construções de produção do conhecimento e contribuições teóricas à pesquisa, enfatizando três scripts epistêmicos, conhecidos como roteiros, que são: evolução, diferenciação e bricolagem. Os autores afirmam que a evolução é uma investigação que orienta os pesquisadores a terem uma visão de melhoria de um trabalho existente. A diferenciação não se baseia em nenhuma investigação; procura-se gerar um conhecimento que é descontínuo com um conhecimento existente, ou seja, é uma investigação nova. E a bricolagem refere-se ao conjunto de diferentes elementos do conhecimento de pesquisa, podendo ter a sua origem em várias disciplinas acadêmicas. Apesar de o Gendron (2008) ter levantado questões do tipo "como se pensa, hoje em dia, a contribuição à pesquisa"? "Como esta última se articula no campo da pesquisa em Contabilidade?" Faz-se necessário refletir sobre esta questão de natureza epistemológica que não suscitou até então muitos estudos no contexto da pesquisa contábil, embora a contribuição seja uma referência central na vida cotidiana. A partir destes fundamentos, parece justificável interessarse aos modelos de contribuição à pesquisa apresentada por Boxenbaum e Rouleau (2011) e estudá-los empiricamente com base nos artigos publicados em Contabilidade Gerencial, uma vez que, cada vez mais, pesquisadores da área vêm conduzindo reflexões sobre o avanço do conhecimento produzido neste campo científico. Portanto, frente a este quadro, surge o seguinte questionamento de pesquisa: Quais são os scripts de produção de conhecimento que influenciam a articulação da contribuição das pesquisas publicadas na área temática de controladoria e contabilidade gerencial dos Congressos Anpcont (Associação Nacional de Programas de Pós-graduação em Ciências Contábeis)?

Para tanto, o objetivo do estudo consiste em analisar o perfil de articulação da contribuição dos artigos da área de Controladoria e Contabilidade Gerencial, de 2010 a 2016, na perspectiva de Boxenbaum e Rouleau (2011). O estudo justifica-se, por um lado, pela carência de pesquisas epistemológicas que analisem os roteiros de produção de conhecimento adotados pelos pesquisadores nessa área temática. E, por outro lado, busca obter evidências empíricas acerca do fenômeno no Anpcont porque é o principal evento que reúne teoricamente pesquisadores dos programas de pós-graduação brasileira, que concentra discussões relativas à Controladoria e Contabilidade Gerencial (CCG) e possibilita o conhecimento de diversos enfoques dos estudos dessa área temática.

Esse artigo contribui com a literatura de várias formas. Primeiro, ele fornece uma análise empírica focada em pesquisas na área de Controladoria e Contabilidade Gerencial, permitindo que os pesquisadores tenham melhor compreensão do direcionamento da articulação das pesquisas contemporâneas em Contabilidade Gerencial sobre contribuição à pesquisa. Segundo, ele evidencia as abordagens paradigmática, teórica e metodológica que servem de base aos scripts de produção de conhecimento que influenciam as pesquisas no campo da Contabilidade Gerencial. Terceiro, o estudo destaca qual (ais) alternativa (s) de script de produção de conhecimento prevalece (em) entre os pesquisadores da comunidade científica da Contabilidade Gerencial, em particular, qual filosofia epistemológica orienta a articulação da pesquisa em Contabilidade Gerencial no tocante à contribuição na geração do conhecimento no Brasil. Por fim, espera-se que o presente trabalho contribua para esclarecer se os scripts de produção de conhecimento adotados pelos pesquisadores brasileiros estão respondendo às críticas levantadas pelos especialistas da área sobre os elementos que dificultam o avanço da geração de conhecimento em Contabilidade Gerencial.

Além desta introdução, o artigo está estruturado da seguinte forma, na seção seguinte, o referencial teórico apresentando as características epistemológicas da pesquisa em Contabilidade Gerencial com ênfase no Brasil e no contexto internacional, a contribuição na pesquisa científica e os três scripts epistêmicos de Boxenbaum e Rouleau (2011) sobre produção de conhecimento no campo organizacional com suas implicações em Contabilidade Gerencial. Em seguida, os procedimentos metodológicos são apresentados. Na seção seguinte, encontram-se a análise e discussão dos resultados. A última seção traz as considerações finais do artigo e destaca as limitações e sugestões para futuras pesquisas. 


\section{Referencial Teórico}

\subsection{Contribuição à pesquisa científica e os três scripts epistêmicos de Boxenbaum e Rouleau (2011)}

De acordo com Berland, Schwarz, Krist, Kenyon, Lo e Reiser (2012) - a contribuição pode ser entendida como o incremento que o artigo fornece aos conhecimentos já adquiridos sobre determinado assunto. Em nenhum caso, esta contribuição se deve ser imperativamente revolucionário. Evidentemente, seria ideal receber contribuições de muita envergadura, mas sabe-se que são muito raras e difíceis. A maioria das contribuições abordam pontos precisos de um corpus teórico e contribuem em fazer avançar de maneira incremental a Ciência Contábil.

A contribuição gera interesse porque enriquece os trabalhos antecedentes e é bem argumentada, clara e precisa. Mais do que uma revolução, a contribuição pode ser uma simples evolução do status do conhecimento. Trata-se então de uma confirmação, inferência, nova proposição. A confirmação é uma contribuição que visa mostrar que proposições antigas, já formuladas antes se revelam ainda válidas. Infelizmente, muitos artigos são proposições de replicações de estudos anteriores, sem grande originalidade. Se a replicação é legítima em ciência, a publicação necessita toda vez que esta replicação se faça com modificações do modelo inicial que apresentam originalidades, permitindo ampliar o campo de aplicação da teoria testada. É preciso que as modificações abordem características cuja natureza pode ser teoricamente intrigante. Replicar um estudo feito nos Estados Unidos, na França, ou na Tunísia é interessante se o autor conseguir mostrar que o contexto institucional coloca a priori uma dúvida sobre a validade da teoria neste contexto (Berland et al., 2012).

A originalidade não é um fim em si. Uma questão pode não ter sido jamais formulada pela simples razão de não ter um grande interesse. Convém então justificar o interesse da questão formulada; um grande número de artigos submetidos peca nesse ponto essencial. O interesse da questão formulada (e da resposta trazida) pode comportar muitos aspectos: gerencial, teórico, metodológico, pedagógico, epistemológico, etc. Existe evidentemente uma grande diversidade de tipos de resultados possíveis para um trabalho de pesquisa, e os revisores estão sempre atentos e abertos a toda a forma de inovação na matéria.

Berland et al. (2012) alertam que, entre as causas mais frequentes de rejeição de artigos na revista "Comptabilité - Contrôle - Audit" figura a insuficiência de contribuição, isto é, incrementos substanciais do artigo ao conhecimento. Um bom artigo deve fazer avançar a ciência. As duas principais vias (às vezes podem ser confundidas) são: a produção de uma contribuição teórica e a produção de novos enunciados para educação. Neste contexto, surgem duas questões fundamentais, a saber, como se pensa atualmente a contribuição na pesquisa? Como ela se articula no campo da pesquisa em Contabilidade, especialmente em Contabilidade Gerencial? É assim que se deve situar estudos visando refletir sobre estas questões de natureza epistemológica que até então não têm estimulado pesquisas no campo da Contabilidade embora a contribuição seja uma referência central na vida cotidiana dos pesquisadores (Gendron, 2013).

Uma das alternativas de se pensar em contribuição foi apresentada por Boxenbaum e Rouleau (2011). Segundo estes pesquisadores, na teoria Organizacional Contemporânea, encontram-se três roteiros epistêmicos que os acadêmicos empregam para conceber e apresentar o novo conhecimento organizacional. Eles são evolução, diferenciação e bricolagem, visualmente representadas na Figura 1. Cada rótulo corresponde a um recurso-chave que separa um script temático dos outros. Eles representam tipos ideais que os estudiosos podem recorrer simultaneamente durante o ato de produção do conhecimento. 


\section{Evolução}

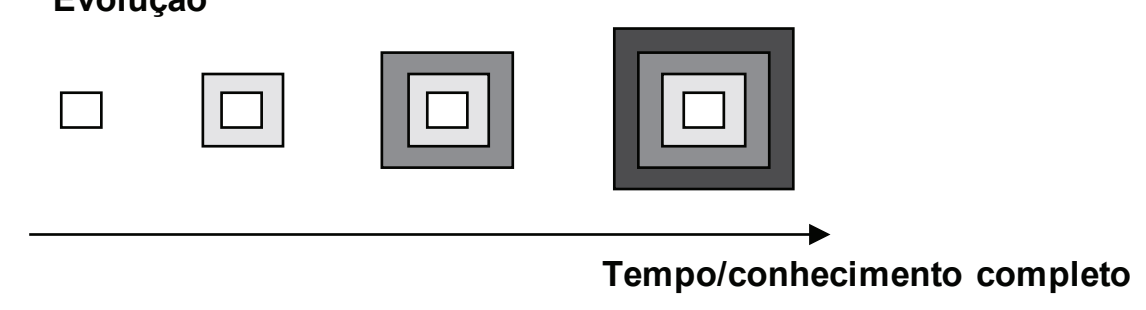

Diferenciação

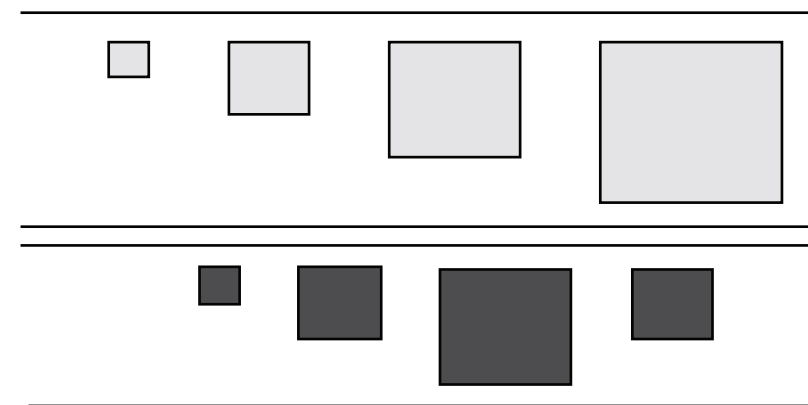

ParadigmaX

Paradigma $Y$

\section{Bricolagem}

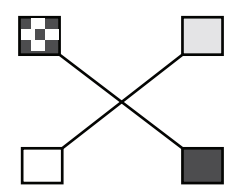

Teoria organizacional A
Teoria organizacional B

Figura 1. Scripts de evolução, diferenciação e bricolagem.

De forma sintetizada, a evolução busca gerar conhecimento a partir de lacunas identificadas em pesquisas anteriores. A diferenciação por meio de rompimento de formas de olhar o objeto de estudo (paradigmas, teorias). A bricolagem é composta a partir de combinação de diferentes paradigmas e teorias, com o intuito de encontrar uma nova luz sobre determinado objeto de estudo. Em relação à evolução, a contribuição é apresentada geralmente no estudo com afirmações contributivas bastante vigorosas e constituídas, como se as ditas contribuições eram definitivamente constituídas de maneira inequívoca. A originalidade nesse tipo de script consiste em resolver, pelo menos parcialmente e temporariamente, um dos enigmas colocados pelo paradigma a que adere o pesquisador (Kuhn, 1983). Quanto ao script de diferenciação, em uma pesquisa, uma "contribuição" será apresentada destacando a distinção, ou seja, explicando como as interpretações e conclusões do estudo são diferentes do corpus estabelecido (Gendron, 2013). Em termos de originalidade, a diferenciação enfatiza a distinção entre os avanços do estudo e os conhecimentos atuais, por meio de mudança de paradigma da pesquisa. Por fim, a bricolagem, segundo Boxenbaum e Rouleau (2011), este script, em vez de inventar uma nova teoria ou novo paradigma, conserta ou remodela teorias existentes, combinando vários conceitos teóricos, ideias e observações em sua disposição imediata. Diante disso, é razoável supor a utilização dos scripts de produção de conhecimento na pesquisa em Contabilidade Gerencial porque é uma àrea de múltiplas perspectivas epistemológicas em que pesquisadores fazem uso de diferentes paradigmas e teorias advindas de outras áreas de conhecimento. 


\subsection{Características epistemológicas e script de produção de conhecimento da pesquisa em Contabilidade Gerencial}

No Brasil, constatou-se, por meio dos estudos epistemológicos da pesquisa em Contabilidade Gerencial que os pesquisadores articulam suas pesquisas para gerar contribuições, apoiando-se em vários paradigmas que orientam suas abordagens teóricas e de método de pesquisa. No entanto, predomina o paradigma funcionalista sobre o paradigma crítico interpretativo, o uso de conceitos contábeis ou legislação sobre as teorias econômica, sociológica ou psicológica em relação às teorias e, pesquisa de campo e levantamentos descritivos sobre trabalhos documentais ou trabalhos de natureza explicativa em relação às estratégias de pesquisa (Nascimento, Junqueira \& Martins, 2010). Essas evidências são ratificadas no estudo de Frezatti et al. (2015) quando revelam que, com base na análise do ano de 2013, os estudos de pesquisadores brasileiros na área de Contabilidade Gerencial são predominantemente quantitativos, muito embora restritos principalmente a uma das possibilidades metodológicas no âmbito quantitativo, deixando de lado outras abordagens que poderiam ser também mais utilizadas nesse âmbito, tais como estudos analíticos, estudos de arquivo e experimentos.

Comparando a pesquisa em Contabilidade Gerencial dentro e fora do Brasil a partir dos eventos científicos europeus e norte-americanos (Conference on New Directions in Management Accounting e Management Accounting Section Midyear Meeting), Frezatti et al. (2015) destacam que enquanto o evento europeu sugere uma predominância de estudos qualitativos, com o emprego do estudo de caso, seguido de perto por levantamentos, como principal abordagem quantitativa, o evento norte-americano apresenta a abordagem quantitativa como a principal e preponderante abordagem de pesquisa, com ênfase ao uso de dados de arquivos e estudos experimentais, em vez de levantamentos, com apenas uma pequena quantidade de estudos com uma abordagem qualitativa.

$\mathrm{Na}$ França, Berland, et al. (2016) investigaram também as características das publicações sobre controle gerencial no principal periódico nacional, "Comptabilité-Contrôle-Audit (CCA)", ao longo de um período de 20 anos e constataram que entre os métodos de pesquisa empregados nessas publicações, predominam revisões da literatura, seguidas de estudos de caso, depois de levantamentos e enfim de entrevistas. Além disso, esses pesquisadores notaram uma desaceleração progressiva de estudos históricos e forte diminuição das revisões de literatura. Em contrapartida, estudos baseados em entrevistas se desenvolveram nos últimos 20 anos. Os métodos quantitativos são ainda pouco explorados nas publicações em controle da revista CCA. Eles são mais utilizados nas revistas internacionais, incluindo a utilização de equações estruturais. Isto sugere que a pesquisa francesa em controle gerencial se baseia fortemente em uma abordagem qualitativa. Ainda, de acordo com os pesquisadores franceses, as bases teóricas para sustentar as pesquisas em controle são ligadas à sociologia, a teoria da Estruturação ou a teoria do Ator Rede e também o construto teórico de sistemas de controle gerencial de Simons.

Além dessas evidências empíricas sobre os aspectos epistemológicos da pesquisa em Contabilidade Gerencial, a comunidade científica da área tem feito algumas críticas epistemológicas que estão à origem da estagnação da produção científica em Contabilidade Gerencial. Elas abrangem as perspectivas paradigmáticas, teórica e metodológica adotada pelos pesquisadores para determinar a forma como a pesquisa deve ser articulada para gerar conhecimento. Com base na nossa análise, constatou-se que as principais reflexões conduzidas atualmente sobre como a pesquisa em contabilidade gerencial deve ser articulada para contribuir com a produção de conhecimento se relacionam com os três (3) modelos de script de produção de conhecimento de Boxenbaum e Rouleau (2011). 
O primeiro script de produção de conhecimento, a evolução, é a perspectiva dominante nas ciências organizacionais para articular a contribuição da pesquisa (Gendron, 2013). Ela tem como alicerce a filosofia de Karl (1959) e assume que os pesquisadores, em uma dada comunidade, estão envolvidos em uma busca comum e avanço gradual do conhecimento. Gendron (2013) acrescenta que a partir desta perspectiva, o objetivo principal, no período chamado de ciência normal por Kuhn (1983) não se destina a questionar o corpo de conhecimento, mas, sim, fortalecer, tijolo por tijolo, peças de um "muro" de conhecimento dedicado a um determinado objeto. A questão de pesquisa específica que estimula o pesquisador é tipicamente associada com a detecção de uma "lacuna" na pesquisa anterior, que Sandberg e Alvesson (2011) chamaram de técnica de gap-spotting. Se a pesquisa em questão é rigorosamente realizada, a lacuna seria pelo menos parcialmente preenchida. Assim, a contribuição resultaria do resultado bemsucedido do teste de erro-tipo ao qual o pesquisador buscou realizar.

Do ponto de vista do script de evolução, pesquisadores em Contabilidade Gerencial têm chamado a atenção sobre a necessidade de uma continuidade nas pesquisas a fim de garantir o estabelecimento do conhecimento produzido. Por exemplo, Shields (2015) afirma que um importante obstáculo que limita a produção de conhecimento estabelecido em Contabilidade Gerencial é o número pequeno de estudos investigando evidências de consistência teórica sobre uma mesma base de variáveis. A pesquisa em Contabilidade Gerencial produzirá mais conhecimento estabelecido se houver mais pesquisas comparativas com resultados consistentes. Quando os resultados de uma pesquisa não são consistentes com os estudos comparativos, isso proporciona oportunidade para conhecer as causas da inconsistência e como elas limitam a produção de conhecimento estabelecido em Contabilidade Gerencial.

Segundo Boxenbaum e Rouleau (2011), o segundo script de produção de conhecimento que pode ser usado para pensar contribuição, é a diferenciação que pressupõe que o mesmo objeto de estudo pode ser pensado de várias as maneiras e examiná-lo a partir de um único ângulo nunca poderá esgotar o potencial de conhecimento inerente a este item. Gendron (2013) menciona que, em outras palavras, construir uma única parede do conhecimento, com base na mesma base teórica, é provável que resulte em contribuições menos importantes e óbvias. Em termos da prática da pesquisa, a diferenciação implica que os investigadores irão submeter-se a produzir obras que estão em descontinuidade com o conhecimento estabelecido (Boxenbaum \& Rouleau 2011).

O segundo script de produção de conhecimento, a diferenciação, baseia-se na premissa de que não há um quadro de referência compartilhado que possa abranger todo o conhecimento acadêmico em relação às organizações. Em vez disso, existem perspectivas diferenciadas que coexistem dentro da teoria Organizacional e que não podem ser ordenadas hierarquicamente (Burrell \& Morgan, 1979; Scherer \& Steinmann, 1999). No contexto da pesquisa em Contabilidade Gerencial, a mudança de paradigma, do positivismo para o interpretativismo e/ou teoria Crítica tem sido destacada por vários pesquisadores como ponto fundamental para que as pesquisas desenvolvidas em Contabilidade Gerencial possam contribuir com o avanço do conhecimento (Frezatti et al., 2015; Hopper \& Bui, 2016). Essa mudança de paradigma reflete o script de diferenciação e a filosofia da ciência associada a Kuhn (1962). Um dos princípios fundamentais dessa filosofia é que a prática da produção do conhecimento se desenvolve dentro de uma comunidade acadêmica que compartilha um conjunto de princípios epistemológicos e suposições ontológicas sobre o mundo, que coletivamente formam o que é conhecido como um paradigma. Um paradigma refere-se a uma base de conhecimento que é mutuamente incomensurável com a de outro paradigma, o que significa que suas suposições fundamentais sobre o mundo e seus modos de produção de conhecimento não podem ser integradas entre si (Scherer \& Steinmann, 1999). 
O terceiro script de produção, a bricolagem, refere-se a uma montagem de elementos prontamente disponíveis. Com esta lente de produção de conhecimento, o pesquisador combina diferentes materiais em mãos para gerar novo conhecimento a partir de um conjunto de intuições e deduções, um ângulo distinto de análise, sintetizando algumas teorias, metáforas e/ou métodos (Boxenbaum \& Rouleau, 2011; Gendron, 2013). Na pesquisa em Contabilidade Gerencial, pesquisadores têm chamado também a atenção sobre a utilização de diferentes paradigmas, teorias e métodos. Por exemplo, Modell (2010) destaca a utilização de métodos mistos e o diálogo por meio de paradigmas múltiplos. Em relação à combinação de teorias, a sua utilização para articular a produção de conhecimento em Contabilidade Gerencial encontra-se ainda muito pouca quando comparada com o uso de uma única teoria nas pesquisas, a exemplo da teoria econômica e dos construtos teóricos de sistemas de controle gerencial (Bromwich \& Scapens, 2016; Frezatti et al., 2015).

Dos três scripts de construção de conhecimento no campo organizacional descritos por Boxenbaum e Rouleau (2011) é difícil dizer se os estudos de Contabilidade Gerencial se enquadram como evolutivos ou diferenciadores ou como um processo de bricolagem. Em boa medida, os ensaios desse campo chamam a atenção sobre pesquisas que busquem complementar lacunas e desenvolver construtos, num processo de desenvolvimento, de ampliação do campo de conhecimento (tipo evolutivo) (Shields, 2015). Por outro lado, há debates no sentido de diferenciar paradigmas, teorias e métodos, utilizando o script de tipo diferenciação (Bromwich \& Scapens, 2016; Frezatti et al., 2015; Modell, 2010).

\section{Procedimentos Metodológicos}

Para realizar a pesquisa, optou-se por analisar os artigos científicos publicados no Congresso Anpcont devido à sua representatividade da comunidade científica brasileira e por representar o fórum de discussão científica dos principais programas de pós-graduação em Contabilidade no país. Além disso, a escolha dessa base se justifica pelo conceito Qualis A do evento junto com a Coordenação de Aperfeiçoamento de Pessoal de Nível Superior (Capes).

Nesse sentido, o presente estudo baseou-se em uma análise descritiva por meio de um levantamento de artigos científicos que foram publicados sobre Controladoria e Contabilidade Gerencial entre 2010 e 2016 em um dos maiores eventos científicos de contabilidade do Brasil, a Anpcont (Associação Nacional dos Programas de Pós-graduação em Ciências Contábeis), a fim de caracterizar e analisar os scripts de produção do conhecimento utilizados pelos pesquisadores brasileiros e também confrontar a tendência dos tipos de contribuição à pesquisa encontrados nas sugestões de Bromwich e Scapens (2016), Frezatti et al. (2015), Shields (2015); Salteiro (2015) a respeito das dificuldades e dos obstáculos no tocante ao avanço do conhecimento em Contabilidade Gerencial.

A pesquisa foi realizada por meio de consultas na base de dados, que fornecem o acesso a artigos científicos publicados nos anais dos congressos anuais: http://www.anpcont.org.br. Os artigos publicados na área temática "Controladoria e Contabilidade Gerencial" foram utilizados para investigar os três roteiros de produção de conhecimentos, conforme ilustrado na Tabela 1. Devido à indisponibilidade de artigos de períodos anteriores a 2010, considerou-se que o estudo de artigos publicados no período de 2010 a 2016 permitiria a aquisição de conhecimentos sobre o estado dos roteiros de produção do conhecimento em Controladoria e Contabilidade Gerencial. 
Tabela 1

\section{Amostra de artigos publicados na área temática no período de 2010 a 2016}

\begin{tabular}{cc}
\hline Ano & $\begin{array}{c}\text { Artigos Publicados } \\
\text { Quantidade }\end{array}$ \\
\hline 2010 & 23 \\
\hline 2011 & 26 \\
\hline 2012 & 28 \\
\hline 2013 & 28 \\
\hline 2014 & 27 \\
\hline 2015 & 32 \\
\hline 2016 & 25 \\
\hline Total & 190 \\
\hline
\end{tabular}

Quanto à seleção dos artigos pesquisas anteriores realizaram estudos tanto epistemológicos, como bibliométricos, com intervalo de tempo semelhante (Cardoso, Pereira \& Guerreiro, 2007; Machado, Nascimento \& Murcia, 2009; Nascimento, Junqueira, \& Martins, 2009; Nascimento, Junqueira \& Martins 2010; Oliveira, 2002, Teóphilo \& Iudícibus, 2009). No período de 2010 a 2016, foram apresentados 190 artigos no referido congresso. Após a elaboração da plataforma teórica, foram definidos os dados que seriam coletados de cada artigo, conforme os argumentos que deram sustentação à questão da pesquisa do ponto de vista epistemológico, isto é, identificar nos artigos argumentos que levam à classificação em função dos três roteiros de produção do conhecimento científico de Boxenbaum e Rouleau (2011) evolução, diferenciação e bricolagem. Os argumentos foram extraídos na base teórica do estudo, definidos em categorias conforme pode ser observado na Figura 2.

\begin{tabular}{|c|c|}
\hline Categorias & Argumentos \\
\hline Evolução & $\begin{array}{l}\text { Apresentação de novas reivindicações de conhecimento como sendo continuidade de } \\
\text { conhecimentos prévios. } \\
\text { Identificação de lacuna em pesquisas anteriores. } \\
\text { Aplicação da técnica gap-spotting. } \\
\text { O produto do conhecimento proposto avança a fronteira do conhecimento. }\end{array}$ \\
\hline Diferenciação & $\begin{array}{l}\text { Apresentação de destaques sobre a distinção, ou seja, a explicação de como as interpretações e } \\
\text { conclusões do estudo são diferentes do corpus estabelecido. } \\
\text { Apresentação de ênfase sobre a distinção entre os avanços do estudo e os conhecimentos } \\
\text { atuais. }\end{array}$ \\
\hline Bricolagem & $\begin{array}{l}\text { Apresentação de um conjunto de intuições e deduções, um ângulo distinto de análise, } \\
\text { sintetizando algumas teorias, metáforas e/ou métodos. } \\
\text { Apresentação de uma nova luz sobre um determinado objeto de estudo, mas a partir de um } \\
\text { ângulo analítico e uma estratégia de investigação com base na nova busca de sentido a partir de } \\
\text { elementos já conhecidos. }\end{array}$ \\
\hline
\end{tabular}

Figura 2. Relação dos argumentos dos roteiros de produção do conhecimento por categoria

Para a classificação dos dados, os pesquisadores fizeram a leitura dos artigos com ênfase na introdução, no referencial teórico, na metodologia e conclusão, especificamente na concepção epistemológica, isto é, na concepção do problema de pesquisa e nas abordagens teóricas e metodológicas. Utilizou-se do software Excel para realizar a tabulação dos dados coletados mediante classificação dos artigos por tema, tipo de contribuição (evolução, diferenciação e bricolagem), quantidade de abordagem teórica e quantidade de método. Os tipos de contribuição foram classificados conforme os três roteiros de produção do conhecimento propostos por Boxenbaum e Rouleau (2011) enquanto que as abordagens teóricas foram classificadas com base na adaptação das teorias adotadas no estudo de Bromwich e Scapens (2016). 


\section{Análise e Discussão dos Resultados}

\subsection{Abordagens paradigmáticas dos artigos científicos em controladoria e contabilidade gerencial publicados no período de 2010 a 2016}

A Tabela 2 evidencia os paradigmas de pesquisa adotados nos artigos científicos publicados na área temática de Controladoria e Contabilidade Gerencial no período de 2010 a 2016. Percebe-se que $97 \%$ do total dos artigos analisados foram classificados no paradigma de pesquisa positivismo e pós-positivismo e, o restante nos paradigmas interpretativismo (1\%) e teoria crítica (2\%) respectivamente.

Tabela 2

Paradigmas de pesquisa dos artigos científicos em Controladoria e Contabilidade Gerencial do congresso Anpcont no período de 2010 a 2016

\begin{tabular}{lcc}
\hline \multicolumn{1}{c}{ Paradigmas de Pesquisa } & Quantidade & Percentual \\
\hline Positivismo e Pós-Positivismo & 185 & $97 \%$ \\
\hline Interpretativismo & 1 & $1 \%$ \\
\hline Interacionismo & - & - \\
\hline Simbólico & - & - \\
\hline Fenomenologia & - & - \\
\hline Hernenêutica & - & $2 \%$ \\
\hline Teoria Crítica & 4 & - \\
\hline Feminismo & - & - \\
\hline Pós-modernismo & - & $100 \%$ \\
\hline Total & 190 & \\
\hline
\end{tabular}

Estes resultados ratificam evidências já mostradas no estudo de Nascimento et al. (2010) onde se revelou que $97 \%$ dos trabalhos abordam a perspectiva funcionalista, ou seja, adotando uma perspectiva positivista. Adicionalmente, constatou-se que os poucos artigos realizados sob o paradigma interpretativo e crítico referem-se a estudos que adotam teorias como a teoria Ator-Rede, teoria da Estruturação e teoria das Representações Sociais. Também, pode-se afirmar que essas evidências se opõem aos resultados encontrados no Conference on New Directions in Management Accounting, em que 49\% dos trabalhos empregam uma ontologia mais à esquerda da matriz de Burell e Morgan (1979), tratando problemas gerenciais pelas óticas interpretativista, estruturalista, pós-estruturalista e de teoria Crítica (Frezatti et al., 2015). Deste modo, pode-se afirmar que as pesquisas publicadas na área temática de Controladoria e Contabilidade Gerencial utilizam de pouca mudança de paradigmas conforme mostrado na Figura 1, para produzir conhecimento sob o script de diferenciação. 


\subsection{Abordagens teóricas dos artigos científicos em Controladoria e Contabilidade Gerencial publicados no período de 2010 a 2016}

Após a descrição e análise dos paradigmas de pesquisa adotados nos artigos científicos publicados no Congresso Anpcont de 2010 a 2016, buscou-se identificar as perspectivas teóricas utilizadas nestes estudos. A Figura 3 apresenta a distribuição das abordagens teóricas conforme a diversidade teórica abordada por Bromwich e Scapens (2016). De acordo com essa mesma figura, constata-se uma predominância de construtos teóricos emanados de sistemas e controle gerencial (34\%) e de teorias econômicas (28\%). Isto mostra também que ainda nas pesquisas da área prevalece o uso de teorias sob a bandeira do paradigma econômico e organizacional. As teorias econômicas mais presentes nos artigos foram teoria da Agência, teoria dos Prospectos, teoria da Utilidade Esperada, teoria Institucional, teoria dos Construtos de Sistemas e controle gerencial foram dos mais variados, por exemplo, modelos alternativos de avaliação de desempenho como Balanced Scorecard. Em seguida, constatou-se a utilização de outras teorias em 20 artigos advindas das teorias organizacionais. Em 13\% dos artigos científicos, não foi possível identificar as teorias utilizadas, nestes casos faltava clareza ou, às vezes mesmo, não havia nenhuma identificada.

\section{Abordagens teóricas dos artigos científicos em controladoria e contabilidade gerencial de 2010 a 2016}

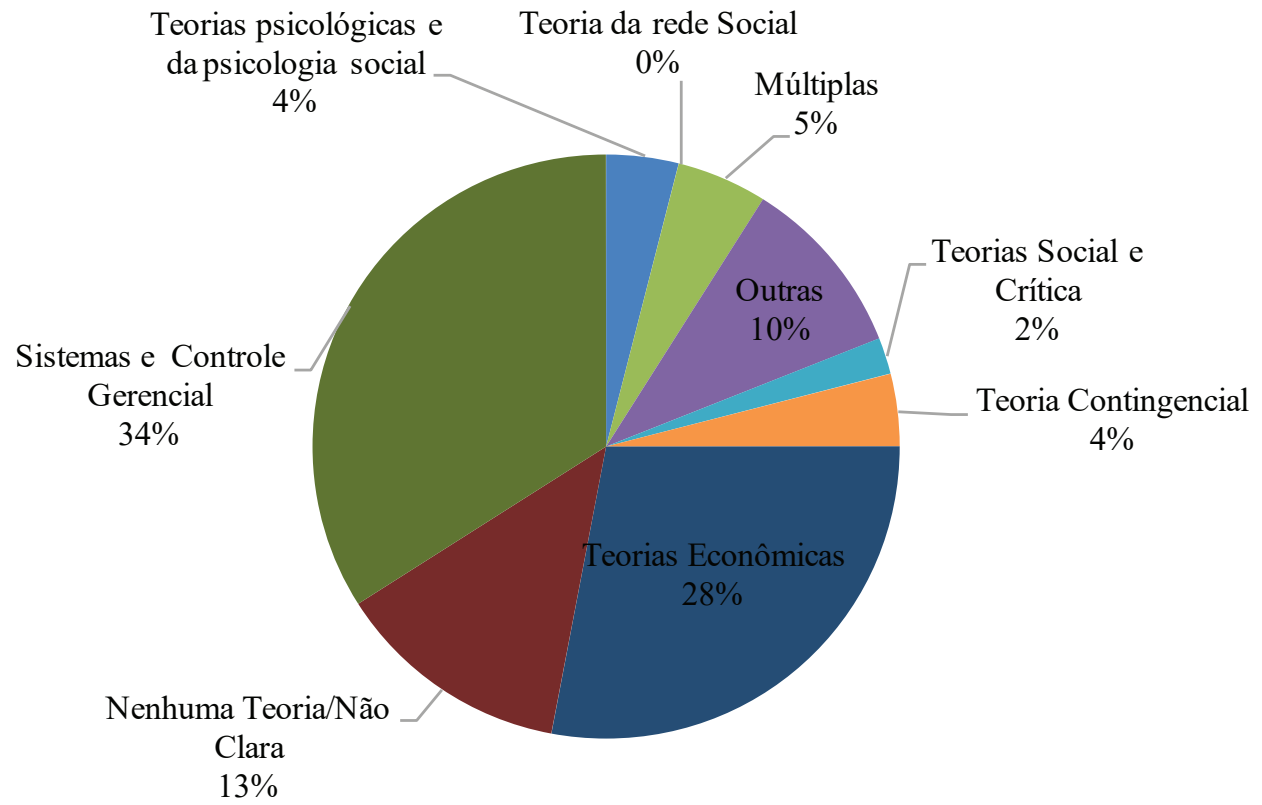

Figura 3. Abordagens teóricas dos artigos científicos no período de 2010 a 2016

Fonte: elaborado pelos autores a partir dos dados da pesquisa

Ainda segundo a Figura 3, enfatiza-se o pequeno número de artigos que utilizaram teoria Social e Crítica (2\%), teoria da Rede Social (0\%) e teorias Múltiplas (5\%), o que traduz que as pesquisas em Controladoria e Contabilidade Gerencial publicadas no Congresso Anpcont, no período de 2010 a 2016, não adotaram perspectivas teóricas sob o paradigma interpretativo e crítico. Além disso, não se verificou a utilização de múltiplas teorias para sustentar uma produção do conhecimento do tipo script de bricolagem. Tais evidências conflitam com as recomendações do periódico europeu Management Accounting Research no sentido de que pesquisas da área devem ampliar as teorias para uma perspectiva interpretativa e pósestruturalista (Hopper \& Bui, 2016). 
Por fim, cabe ressaltar a pouca utilização de teorias Psicológica e Psicologia Social (4\%) e da teoria Contingencial (4\%). Mais uma vez, em relação a esta última, que de acordo com Bromwich e Scapens (2016), a pesquisa contábil gerencial tem apresentado um crescimento de teorias Social e Crítica.

\subsection{Abordagens de métodos de pesquisa dos artigos científicos em Controladoria e Contabilidade Gerencial publicados no período de 2010 a 2016}

A Figura 4 apresenta o perfil das abordagens de métodos de pesquisa dos artigos científicos publicados no Congresso Anpcont de 2010 a 2016. De acordo com a mesma Figura 4, observa-se que 64\% dos trabalhos pesquisados utilizaram como abordagem de método de pesquisa o quantitativo, confirmando assim o método adotado pelo mainstream da pesquisa em Contabilidade Gerencial. Vale salientar que os trabalhos de natureza qualitativa foram $18 \%$ do total dos artigos e o restante distribuído em trabalhos quali-quantitativo (13\%) e não empírico (5\%). Estes resultados corroboram a análise de Frezatti et al. (2015) sobre o estágio da pesquisa em Contabilidade Gerencial no Brasil. Existe uma predominância de estudos quantitativos na pesquisa em Contabilidade Gerencial, especificamente por meio de levantamentos. Adicionalmente, quando se observa a dimensão qualitativa, o emprego de estudos de caso tem sido o mais utilizado por pesquisadores em Contabilidade Gerencial. A predominância desses dois métodos está em consonância com o identificado por Nascimento et al. (2010), que constataram esses dois métodos como os mais comuns nos principais eventos acadêmicos da área de Contabilidade (incluindo o Encontro da Associação Nacional de Pós-Graduação e Pesquisa em Administração - Enanpad), no período de 2005-2008.

\section{Perfil das Abordagens de método de Pesquisa}

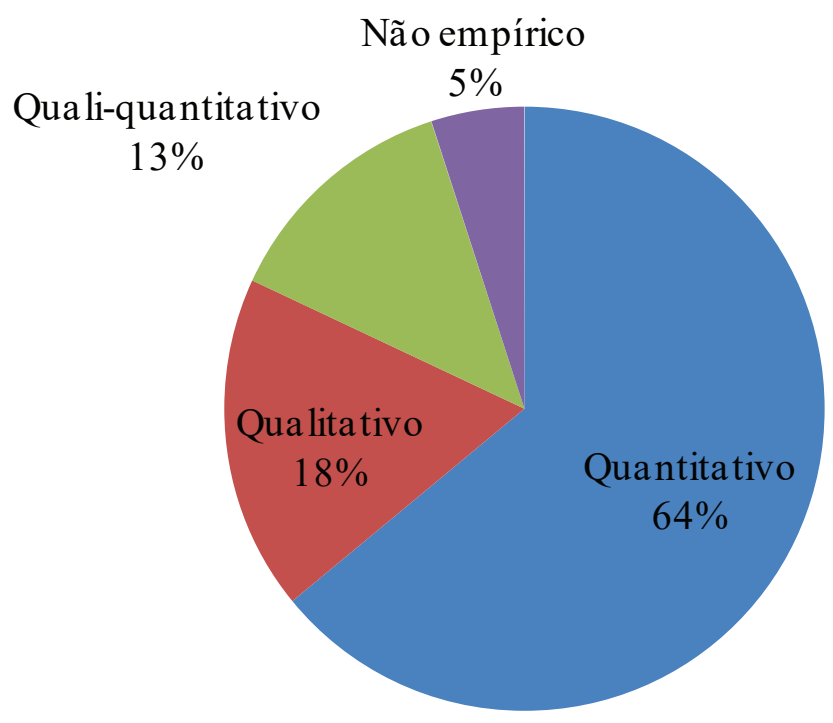

Figura 4. Perfil das abordagens de método de pesquisa dos artigos científicos publicados no Congresso Anpcont de 2010 a 2016

Além disso, nota-se um ligeiro crescimento na utilização do método quali-quantitativo que se alinha ao padrão metodológico das pesquisas internacionais em Contabilidade Gerencial, onde estas buscam, cada vez mais, integrar as pesquisas quantitativas e qualitativas. Neste sentido, os estudos realizados no Brasil e publicados no Anpcont estão corroborando a tese de Modell (2010), que destacou que os métodos mistos estão sendo amplamente examinados no contexto da pesquisa de Contabilidade Gerencial e o diálogo por meio de paradigmas múltiplos recebeu atenção por parte dos pesquisadores brasileiros. 


\subsection{Classificação dos artigos científicos em Controladoria e Contabilidade Gerencial por tipo de contribuição}

A Tabela 3 apresenta a quantidade de artigos científicos em Controladoria e Contabilidade Gerencial por tipo de script de produção de conhecimento. Constata-se que do total de 190 artigos publicados na Anpcont, no período de 2010 a 2016, 163 artigos foram classificados no grupo do script de produção de conhecimento do tipo evolução, representando aproximadamente $86 \%$ do total. Em seguida, tem-se 22 artigos científicos classificados no script de produção de conhecimento do tipo bricolagem, equivalendo a $11 \%$ da quantidade total de artigos. Em relação ao script de diferenciação, percebe-se também por meio da mesma Tabela 3 que houve um número pequeno de artigos com este tipo de contribuição. Diante desses dados, pode-se observar inicialmente que, em geral, a produção de conhecimento em Controladoria e Contabilidade Gerencial no período estudado mostra uma perspectiva predominante de contribuição do tipo evolução. Além disso, cabe ressaltar também um pequeno número de trabalhos buscando produzir conhecimentos através do script tipo bricolagem.

Tabela 3

Frequência dos scripts de produção de conhecimento no período total

\begin{tabular}{lcc}
\hline Scripts de Produção de Conhecimento & Quantidade & Percentual \\
\hline Evolução & 163 & $86 \%$ \\
\hline Diferenciação & 5 & $3 \%$ \\
\hline Bricolagem & 22 & $11 \%$ \\
\hline Total & 190 & $100 \%$ \\
\hline
\end{tabular}

Como pode ser observado na Tabela 3, ao longo do período analisado, constatou-se que dos $86 \%$ dos artigos adotaram o script de evolução, no período de 2010 a 2016. Mais de $80 \%$ dos artigos publicados anualmente adotam o script de evolução chegando a uma frequência de 96\%, no ano de 2013, o que mostra uma predominância desta perspectiva de contribuição nos artigos publicados no Congresso Anpcont. Além disso, percebe-se também certa estabilidade no uso deste script de produção de conhecimento ao longo do período analisado.

Tabela 4

Frequência dos tipos de contribuição por ano

\begin{tabular}{ccccccccc}
\hline \multirow{2}{*}{ ANO } & \multicolumn{7}{c}{ TIPOS DE CONTRIBUIÇÃO DA PESQUISA } & \multicolumn{1}{c}{$c$} \\
\cline { 2 - 10 } & EVOLUÇÃO & $\%$ & DIFERENCIAÇÃO & $\%$ & BRICOLAGEM & $\%$ & TOTAL & $\%$ \\
\hline 2010 & 19 & $83 \%$ & 1 & $4 \%$ & 3 & $13 \%$ & 23 & $100 \%$ \\
\hline 2011 & 22 & $85 \%$ & 2 & $7,5 \%$ & 2 & $7,5 \%$ & 26 & $100 \%$ \\
\hline 2012 & 24 & $86 \%$ & - & - & 4 & $14 \%$ & 28 & $100 \%$ \\
\hline 2013 & 27 & $96 \%$ & - & - & 1 & $4 \%$ & 28 & $100 \%$ \\
\hline 2014 & 24 & $89 \%$ & - & - & 3 & $11 \%$ & 27 & $100 \%$ \\
\hline 2015 & 25 & $78 \%$ & 1 & $3 \%$ & 6 & $19 \%$ & 32 & $100 \%$ \\
\hline 2016 & 21 & $84 \%$ & 1 & $4 \%$ & 3 & $12 \%$ & 25 & $100 \%$ \\
\hline TOTAL & 163 & $88 \%$ & 5 & $3 \%$ & 23 & $11 \%$ & 190 & $100 \%$ \\
\hline
\end{tabular}


Em relação ao script de bricolagem, constataram-se frequências relativas abaixo de $20 \%$ do total de artigos publicados em Controladoria e Contabilidade Gerencial em cada edição do Congresso. Isto revela que a produção científica desta área de conhecimento não segue esta perspectiva de contribuição à pesquisa para gerar conhecimento no campo da Controladoria e Contabilidade Gerencial. No tocante ao script de diferenciação, o período de 2011 apresentou um percentual aproximadamente de $8 \%$, o que traduz que poucos artigos foram classificados neste tipo de contribuição, o que mostra que os trabalhos publicados e analisados em Controladoria e Contabilidade Gerencial não buscam se diferenciar uns aos outros para gerar novo conhecimento para a área. Em resumo, pode-se destacar, a partir da análise descritiva, feita sobre o diagnóstico dos scripts de produção de conhecimento nos artigos publicados em Controladoria e Contabilidade Gerencial, que há uma predominância de utilização da perspectiva do script de evolução. Tal evidência confirma a utilização da perspectiva de contribuição mais comumente pela comunidade científica no geral nas pesquisas da área temática de controladoria e contabilidade gerencial do Congresso Anpcont.

\subsection{Evolução dos tipos de contribuição dos artigos científicos em função do período estudado}

Como o principal objetivo desse estudo consistiu em analisar a perspectiva dominante da contribuição à pesquisa em Controladoria e Contabilidade Gerencial, faz-se necessário analisar o comportamento evolutivo dos scripts de produção de conhecimento ao longo do período de estudo. A Figura 6 mostra a evolução dos três scripts de produção de conhecimento da área temática de Controladoria e Contabilidade Gerencial das edições do Congresso Anpcont, no período de 2010 a 2016. No geral, como pode ser constatado no mesmo gráfico, o script de evolução se sobrepõe aos demais scripts, de diferenciação e bricolagem.

\section{Evolução dos Tipos de scripsts de produção de conhecimento em Controladoria e Contabilidade Gerencial de 2010 a 2016}

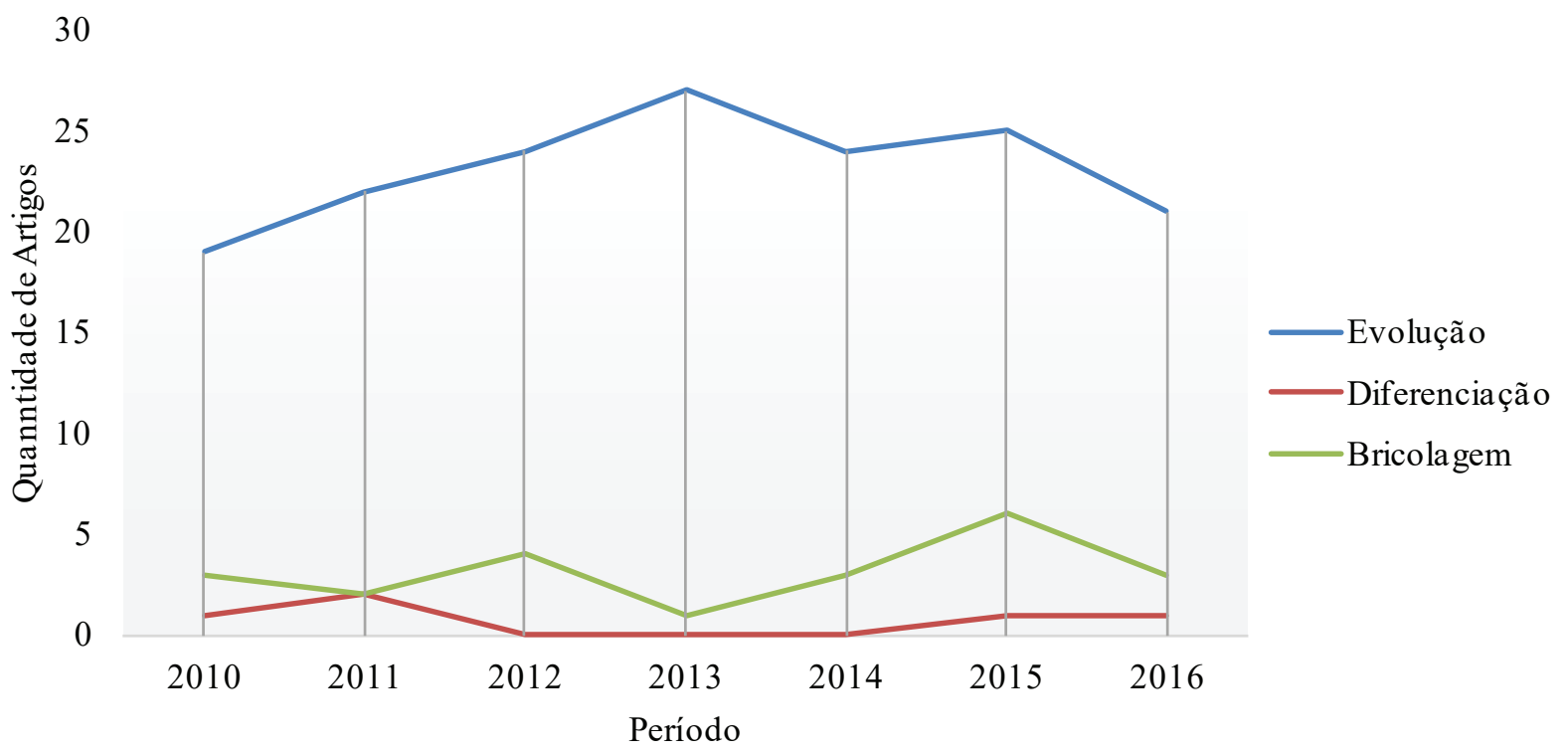

Figura 6. Evolução dos scripts de produção de conhecimento em Controladoria e Contabilidade Gerencial no período de 2010 a 2016 
No primeiro subperíodo, de 2010 a 2013, observa-se que um crescimento do uso do script de evolução nas pesquisas nesta área temática enquanto o uso do script de bricolagem permanece constante nos dois primeiros anos (2010 e 2011) equivalendo aproximadamente de $13 \%$ do total dos artigos publicados em cada edição. No período de 2011 a 2012, observa-se um aumento (14\%) e depois um declínio de 2012 a 2013 (4\%) na utilização deste script. O crescimento na utilização do script de bricolagem ficou mais acentuado nas edições de 2014 e 2015. Quanto ao segundo subperíodo, de 2013 a 2016, percebeu-se através do Gráfico 3 um aumento da utilização do script de bricolagem até a edição de 2015 contra um declínio seguido de oscilação do uso do script de evolução pelos pesquisadores em Controladoria e Contabilidade Gerencial. Cabe ressaltar aqui a utilização do script de bricolagem em 6 artigos (19\%) no ano de 2015, o que traduz um interesse cada vez maior por pluralismo paradigmático, teórico ou metodológico embora ainda seja insignificante diante da predominância do script de evolução.

\section{Considerações Finais}

Este trabalho buscou avaliar o progresso da pesquisa em Contabilidade Gerencial no geral definida na área temática em um dos dois maiores eventos de Contabilidade do Brasil. Adicionalmente, buscouse conduzir esta discussão à luz das recentes publicações de editoriais de duas renomadas revistas internacionais especializadas da área, Management Accounting Research e Journal of Management Accounting Research (JMAR) especificamente dedicadas ao desenvolvimento da pesquisa em Contabilidade Gerencial. Portanto, com base no estudo realizado, verificou-se que a perspectiva dominante da pesquisa na linha temática de Controladoria e Contabilidade Gerencial é do tipo evolução, mostrando que a pesquisa da área segue a perspectiva da filosofia de $\operatorname{Karl}$ (1959) e, assume os pesquisadores, na comunidade brasileira, estão envolvidos em uma busca comum e avanço gradual do conhecimento. Este resultado pode ser enquadrado também na perspectiva da ciência normal de Kuhn (1983), quando afirmou que o script evolutivo não se destina a questionar o corpo de conhecimento, mas, sim, fortalecer, tijolo por tijolo, peças de um "muro" de conhecimento dedicado a um determinado objeto. Em outras palavras, pode-se afirmar que a forma de articulação utilizada pelos pesquisadores da área de Contabilidade Gerencial no Brasil corresponde à detecção de uma "lacuna" na pesquisa anterior, o que Sandberg e Alvesson (2011) chamaram de técnica de gap-spotting.

Esta evidência confirma ainda a predominância do paradigma positivista e a incipiência do uso de paradigmas alternativos tais como o interpretativismo e a teoria Crítica. Assim, as pesquisas publicadas na área temática de Controladoria e Contabilidade Gerencial utilizam-se de pouca mudança de paradigmas para produzir conhecimento sob o script de diferenciação. Apesar de os resultados mostrarem uma evolução lenta da utilização desse roteiro de produção de conhecimento nos últimos anos e os resultados mostraram que pesquisas da Contabilidade Gerencial no Brasil têm buscado alternar paradigmas para investigar um mesmo objeto de pesquisa.

Sobre as abordagens teóricas, há uma predominância de construtos teóricos emanados de sistemas e controle gerencial e de teorias econômicas. Isto mostra também que ainda nas pesquisas da área prevalece o uso de um único paradigma baseado na teoria econômica. No estudo, foi enfatizado também um pequeno número de artigos que utilizaram teoria Social e Crítica, teoria da Rede Social e teorias Múltiplas, o que traduz que as pesquisas em controladoria e contabilidade gerencial publicadas no Congresso Anpcont, no período de 2010 a 2016, não adotaram perspectivas teóricas sob o paradigma interpretativo e crítico. Além disso, não se verificou a utilização de múltiplas teorias para sustentar uma produção do conhecimento do tipo script de bricolagem. Tais evidências conflitam com as recomendações do periódico europeu Management Accounting Research no sentido de que pesquisas da área devem ampliar as teorias para uma perspectiva interpretativa e pós-estruturalista (Hopper \& Bui, 2016). A teoria Contingencial apresentada por Bromwich e Scapens (2016) como a mais utilizada para obter conhecimento estabelecido em contabilidade, foi pouco utilizada nos trabalhos. 
No tocante ao perfil das abordagens de método de pesquisa dos artigos científicos publicados no Congresso Anpcont, de 2010 a 2016. Os resultados revelaram uma predominância de estudos quantitativos na pesquisa em Contabilidade Gerencial, especificamente por meio de levantamentos. Na dimensão qualitativa, o emprego de estudos de caso tem sido o mais utilizado por pesquisadores em contabilidade gerencial. Adicionalmente, constatou-se um crescimento insignificativo na utilização do método qualiquantitativo adotado como padrão metodológico das pesquisas europeias em Contabilidade Gerencial, onde estas buscam, cada vez mais, integrar as pesquisas quantitativas e qualitativas (Lukka, 2010; Modell, 2010).

Por fim, o presente estudo apresenta limitações quanto à classificação dos artigos conforme as abordagens epistemológicas, teóricas, metodológicas e de script de produção de conhecimento. Também, os resultados restringem aos artigos publicados no banco de dados da Anpcont e no período analisado no estudo. Como sugestões para futuras pesquisas, recomenda-se que estudos busquem, por exemplo, analisar as perspectivas dominantes de script de produção por tema para que se possa identificar quais estudos estão sendo consolidados para obter geração de conhecimento na área (Hesford et al., 2006; Otley, 2016).

\section{Referências}

Araújo, E. A. T., \& Silva, W. A. C. (2010). Pesquisa científica em contabilidade gerencial nos Enanpads de 2003 a 2008. Revista Universo Contábil, 6(3), pp. 29-44.

doi: https://doi.org/10.4270/ruc.2010320

Berland, L. K., Schwarz, C. V., Krist, C., Kenyon, L., Lo, A. S. \& Reiser, B. J. (2016). Epistemologies in practice: Making scientific practices meaningful for students. Journal of Research in Science Teaching, 53(7), pp. 1082-1112.

doi: https://doi.org/10.1002/tea.21257

Berland, N., Stolowy, H. \& Piot, C. (2012). Éditorial. Qu>est-ce qu>une «bonne» contribution?. ComptabilitéContrôle-Audit, 18(2), pp. 3-6.

doi: https://doi.org/10.3917/cca.182.0003

Berry, A. J., Coad, A. F., Harris, E. P., Otley, D. T. \& Stringer, C. (2009). Emerging themes in management control: A review of recent literature. The British Accounting Review, 41(1), pp. 2-20. doi: https:// doi.org/10.1016/j.bar.2008.09.001

Boxenbaum, E. \& Rouleau, L. (2011). New knowledge products as bricolage: Metaphors and scripts in organizational theory. Academy of Management Review, 36(2), pp. 272-296.

doi: https://doi.org/10.5465/amr.2011.59330898

Bromwich, M. \& Scapens, R. W. (2016). Management Accounting Research: 25 years on. Management Accounting Research, 31, pp. 1-9. doi: https://doi.org/10.1016/j.mar.2016.03.002

Burell, G. \& Morgan, G. (1979). Sociological paradigms and organisational analysis. Elements of the sociology of corporate life.

Cardoso, R. L., Pereira, C. A. \& Guerreiro, R. (2007). Perfil das pesquisas em contabilidade de custos apresentadas no EnANPAD no período de 1998 a 2003. Revista de Administração Contemporânea, 11(3), pp. 177-198. doi: https://doi.org/10.1590/s1415-65552007000300009

Croom, S. (2001). The dyadic capabilities concept: examining the processes of key supplier involvement in collaborative product development. European Journal of Purchasing and Supply Management, 7 (1), pp. 29 - 37. doi: https://doi.org/10.1016/s0969-7012(00)00019-8 
Cruz, A. P. C. D., Costa, F., Espejo, M. M. D. S. B. \& Almeida, L. B. D. (2011). Redes de cooperação entre pesquisadores no congresso USP de controladoria e contabilidade: uma análise retrospectiva do período 2001-2009. 22(55), pp. 64-87

Frezatti, F., Aguiar, A. B. de Araújo Wanderley, C. de \& Malagueño, R. (2015). A pesquisa em contabilidade gerencial no Brasil: desenvolvimento, dificuldades e oportunidades. Revista Universo Contábil, 11(1), pp. 47-68. doi: https://doi.org/10.4270/ruc.2015103

Frezatti, F., Nascimento, A. R. D. \& Junqueira, E. R. (2008). Demandas metodológicas, monoparadigma e o desenvolvimento da contabilidade gerencial. Anais do Congresso da Associação Nacional de Programas de Pós-Graduação em Ciências Contábeis. Salvador, BA, Brasil, 2

Gendron, Y. (2008). Constituting the academic performer: the spectre of superficiality and stagnation in academia. European Accounting Review, 17(1), pp. 97-127.

doi: https://doi.org/10.1080/09638180701705973

Gendron, Y. (2013). Rethinking the concept of research contribution. Comptabilité-Contrôle-Audit, 19(2), pp. 133-155. doi:https://doi.org/10.3917/cca.192.0133

Helford, J. W., Lee, S. H. S., Van der Stede, W. A. \& Young, S. M. (2006). Management accounting: a bibliographic study. Handbooks of management accounting research, 1, pp, 3-26. doi: https://doi. org/10.1016/s1751-3243(06)01001-7

Hopper, T. \& Bui, B. (2016). Has management accounting research been critical?. Management Accounting Research, 31, pp. 10-30.

doi: https://doi.org/10.1016/j.mar.2015.08.001

Karl, P. (1959). The logic of scientific discovery. Hutchinson, Londres.

Kuhn, T. S. (1962). The Structure of Scientific Revolutions. Chicago (University of Chicago Press) 1962. doi: https://doi.org/10.1086/ahr/68.3.700

Kuhn, T. S. \& Meyer, L. (1983). La structure des révolutions scientifiques.

Lukka, K. (2010). The roles and effects of paradigms in accounting research. Management Accounting Research, 21(2), pp. 110-115. doi: https://doi.org/10.1016/j.mar.2010.02.002

Lunkes, R. J., Ripoll Feliu, V. M. \& Rosa, F. S. (2011). Redes sociais e internacionalização da contabilidade gerencial: um estudo em publicações de língua espanhola Congresso de Contabilidade e Auditoria, Porto, Portuga, pp. 1-15.

Machado, M. R., Nascimento, A. R. D. \& Murcia, F. D. (2009). Análise crítica-epistemológica da produção científica em contabilidade social e ambiental no Brasil. Congresso USP de Controladoria e Contabilidade. São Paulo, SP, Brasil, 9.

Modell, S. (2010). Bridging the paradigm divide in management accounting research: The role of mixed methods approaches. Management Accounting Research, 21(2), pp. 124-129.

doi: https://doi.org/10.1016/j.mar.2010.02.005

Nascimento, A. R. D., Junqueira, E. \& Martins, G. D. A. (2009). Análise epistemológica da produção científica em contabilidade gerencial no Brasil. Encontro Anual da Associação Nacional dos Programas de Pós-graduação em Administração - Enanpad, Salvador, BA, Brasil, 33

Nascimento, A. R. , Junqueira, E. \& Martins, G. A. (2010). Pesquisa acadêmica em contabilidade gerencial no Brasil: análise e reflexões sobre teorias, metodologias e paradigmas. Revista de Administração Contemporânea, 14(6), pp. 1113-1133.

doi: https://doi.org/10.1590/s1415-65552010000700008 
Oliveira, M. C. (2002). Análise dos periódicos brasileiros de contabilidade. Revista Contabilidade \& Finanças, 13(29), pp. 68-86. doi: https://doi.org/10.1590/s1519-70772002000200005

Otley, D. (2016). The contingency theory of management accounting and control: 1980-2014. Management accounting research, 31, pp. 45-62. doi:: https://doi.org/10.1016/j.mar.2016.02.001

Salterio, S. E. (2015). Barriers to knowledge creation in management accounting research. Journal of Management Accounting Research, 27(1), pp. 151-170. Doi: https://doi.org/10.2308/jmar-51056

Sandberg, J. \& Alvesson, M. (2011). Ways of constructing research questions: gap-spotting or problematization?. Organization, 18(1), pp. 23-44. Doi: https://doi.org/10.1177/1350508410372151

Scapens, R. W. \& Bromwich, M. (2001). Editorial report-management accounting research: The first decade. Management accounting research, 12(2), pp. 245-254. Doi: https://doi.org/10.1006/ mare.2001.0159

Scapens, R. W. \& Bromwich, M. (2010). Management accounting research: 20 years on. Management Accounting Research, 21(4), pp. 278-284. doi: https://doi.org/10.1016/j.mar.2010.08.003

Scherer, A. G. \& Steinmann, H. (1999). Some remarks on the problem of incommensurability in organization studies. Organization Studies, 20(3), pp. 519-544. doi: https://doi.org/10.1177/0170840699203006

Shields, M. D. (2015). Established management accounting knowledge. Journal of Management Accounting Research, 27(1), pp. 123-132. Doi: https://doi.org/10.2308/jmar-51057

Theóphilo, C. R. \& Iudícibus, S. (2009). Uma análise crítico-epistemológica da produção científica em contabilidade no Brasil. Revista Contabilidade, Gestão e Governança, 8(2). 